\title{
Estradiol Acetate
}

National Cancer Institute

\section{Source}

National Cancer Institute. Estradiol Acetate. NCI Thesaurus. Code C47990.

The acetate salt form of estradiol, the most potent, naturally produced estrogen.

Estradiol acetate diffuses through the cell membrane and binds to and subsequently activates the nuclear estrogen receptor found in the reproductive tract, breast, pituitary, hypothalamus, liver, and bone. The activated complex binds to the estrogen response element on the DNA and activates the transcription of genes involved in the functioning of the female reproductive system and secondary sex characteristics. 